\title{
Desarrollo y validación de la versión breve del cuestionario de motivos de consumo de marihuana (MMM SF)
}

\section{Development and Validation of the Marijuana Motives Measure Short Form}

\author{
Laura Mezquita***, Lucía Ruiz-Valero*, Naiara Martínez-Gómez*, Manuel I. Ibáñez****, \\ Generós Ortet*,**. \\ *Departamento de Psicología Básica, Clínica y Psicobiología, Universitat Jaume I, Castellón, España. \\ **Centro de Investigación Biomédica en Red, Salud Mental (CIBERSAM), Instituto de Salud Carlos III, Madrid, España.
}

\section{Resumen}

Los motivos de consumo son una variable proximal al uso de marihuana. Este estudio pretende adaptar y validar la versión española breve del Marijuana Motives Measure (MMM; Simons, Correia, Carey, y Borsari, 1998), el MMM SF.

La muestra estaba compuesta por 232 participantes $($ edad media $=$ 25,11 (7,58), 50,43\% hombres) que habían probado la marihuana al menos una vez. Se realizaron análisis de los ítems y de Rasch para seleccionar los 15 ítems. El Análisis Factorial Confirmatorio (AFC) mostró una estructura de cinco factores adecuada $\left({ }_{S-B} X^{2}(80)=121,30\right.$, $p=, 002 ; \mathrm{NNFI}=0,944 ; \mathrm{CFI}=0,958 ; \mathrm{IFI}=0,959 ; \mathrm{MFI}=0,915 ; \mathrm{RMSEA}=$ 0,047 (0,029, 0,063); AIC = -38,70), y el AFC multigrupo entre hombres y mujeres mostró índices de ajuste aceptables $\left(_{{ }_{S B}} X^{2}(160)=230,01, p\right.$ $=, 000 ; \mathrm{NNFI}=0,900 ; \mathrm{CFI}=0,924 ; \mathrm{IFI}=0,927 ; \mathrm{MFI}=0,860 ; \mathrm{RMSEA}=$ 0,062 (0,043, 0,078); AIC = -89,99). El cuestionario mostró invarianza métrica $\left.\left({ }_{S B B} X_{d i f}^{2}(15)=13,61, p=, 556\right)\right)$, escalar $\left({ }_{S \cdot B} X_{d i f}^{2}(15)=23,15, p=\right.$ $, 081)$ ) y de los errores de medida $\left.\left({ }_{s \cdot B} X_{d i f}^{2}(15)=8,65, p=, 895\right)\right)$ entre grupos de género. Los alfas de Cronbach y omega ordinal de las escalas fueron de 0,79 a 0,90. Los motivos de animación, afrontamiento y bajos motivos de conformidad predijeron el consumo de marihuana. El mejor predictor durante la época de mayor consumo fueron los motivos de animación, mientras que los motivos de afrontamiento, y en menor medida los bajos motivos de conformidad, fueron los mejores predictores de los problemas derivados una vez se controló el efecto de frecuencia y cantidad fumada.

El MMM SF presenta unas propiedades psicométricas adecuadas y es una medida útil para evaluar los motivos de consumo de marihuana, especialmente durante sesiones de evaluación con tiempo limitado. Palabras clave: Motivos de consumo de marihuana; Cannabis; MMM SF; Propiedades psicométricas; Variables de consumo de marihuana.

\begin{abstract}
Marijuana motives are a proximal variable to marijuana use. This research aimed to adapt and validate the short form of the Marijuana Motives Measure (MMM; Simons, Correia, Carey, y Borsari, 1998), the MMM SF, in Spanish.

The sample comprised 232 participants (mean age $=25.11(7.58)$, $50.43 \%$ males) who had tried marijuana at least once in their lifetime. Item and Rasch analyses were performed to choose the final pool of 15 items. Confirmatory Factor Analysis (CFA) showed an adequate 5-factor structure $\left({ }_{s \cdot B} X^{2}(80)=121.30, p=.002 ; \mathrm{NNFI}=.944 ; \mathrm{CFI}\right.$ $=.958 ; \mathrm{IFI}=.959 ; \mathrm{MFI}=.915 ; \mathrm{RMSEA}=.047(0.029,0.063) ; \mathrm{AIC}=$ -38.70), and the multi-group CFA between males and females showed acceptable fit indices $\left({ }_{S B} X^{2}(160)=230.01, p=.000 ; \mathrm{NNFI}=.900\right.$; $\mathrm{CFI}=.924 ; \mathrm{IFI}=.927 ; \mathrm{MFI}=.860 ; \mathrm{RMSEA}=.062(.043, .078) ; \mathrm{AIC}=$ -89.99). The questionnaire indicated metric $\left({ }_{S \cdot B} X_{\text {diff }}^{2}(15)=13.61, p=\right.$ $.556)$ ), scalar $\left.\left({ }_{S \cdot B} X_{\text {diff }}^{2}(15)=23.15, p=.081\right)\right)$ and error measurement invariance $\left.\left({ }_{s-B} X_{d i f f}^{2}(15)=8.65, p=.895\right)\right)$ between gender groups. The internal consistencies and ordinal omega of the scales were between .79 and .90. In the regression analysis, enhancement, coping and low conformity motives predicted frequency and quantity of marijuana smoked. The best predictor of frequency and quantity consumed during the heaviest smoking period was enhancement, while coping and, to a lesser extent, low conformity, were the only predictors of cannabis-related problems when marijuana frequency and quantity were controlled for.

The MMM SF shows adequate psychometric properties and is a suitable instrument to assess marijuana motives, especially during time-limited sessions.

Keywords: Marijuana motives; Cannabis; MMM SF; Psychometric properties; Marijuana outcomes.
\end{abstract}

Recibido: Junio 2017; Aceptado: Septiembre 2017

Enviar correspondencia a:

Laura Mezquita. Department of Basic and Clinical Psychology and Psychobiology, Universitat Jaume I, Av. de Vicent Sos Baynat, s/n, 12071 Castelló, Spain. E-mail: lmezquit@uji.es. 
E 1 cannabis (marihuana) es la droga ilegal más ampliamente consumida a nivel mundial (UNODC, 2015). En 2013, alrededor de 181,8 millones de personas (con márgenes de incertidumbre entre 128,5232,1 millones) de entre 15 y 64 años consumía cannabis con fines no medicinales (UNODC, 2015). El cannabis perjudica diversas funciones cognitivas de forma aguda, y su consumo se asocia con sufrir accidentes de tráfico bajo los efectos de la droga, y con síntomas cardiovasculares y psicóticos, entre otros (OMS, en prensa). Por estas razones, es importante prevenir y reducir el consumo de cannabis; para logarlo, es esencial saber por qué las personas fuman esta droga.

El Marijuana Motives Measure (MMM; Simons et al., 1998) es uno de los cuestionarios más ampliamente utilizados para evaluar los motivos. El MMM fue desarrollado por Simons et al. (1998), y se basa en el Drinking Motives Questionnaire-Revised (DMQ-R) desarrollado por Cooper (1994) para evaluar las razones por las cuáles las personas beben alcohol. Como resultado, el MMM (Simons et al., 1998) se compone de cuatro motivos de consumo de marihuana, basados en (a) el tipo de refuerzo deseado (refuerzo positivo o negativo) y (b) la fuente del refuerzo (interna o externa). Del cruce de estas dos dimensiones surgen cuatro motivos diferentes de consumo de marihuana: los motivos sociales (externo, positiva) hacen referencia al acto de fumar marihuana para facilitar las relaciones sociales; los motivos de animación (interno, positiva) hacen referencia al uso de cannabis para aumentar el afecto positivo; los motivos de conformidad (externo, negativa) se refieren a fumar con la intención de integrarse en un grupo de iguales; y los motivos de afrontamiento (interno, negativa) hacen referencia a fumar cannabis para manejar el afecto negativo. Simons et al. (1998) también añadieron un quinto motivo de consumo de marihuana, los motivos de expansión, que hacen referencia al uso de cannabis para ser más creativo y original, comprender las cosas de manera diferente, y ser más abierto a experiencias. Tanto los análisis factoriales exploratorios (Chabrol, Ducongé, Casas, Roura, y Carey, 2005; Simons et al., 1998) como los análisis factoriales confirmatorios (Zvolensky et al., 2007) han apoyado esta estructura del cuestionario de cinco factores. Las cinco escalas resultantes mostraron buenas consistencias internas con alfas de Cronbach de 0,70 o superior (Chabrol et al., 2005; Simons et al., 1998; Zvolensky et al., 2007). No obstante, estudios recientes realizados con el MMM han encontrado que algunas de las cargas factoriales de los ítems originales eran inapropiadas (Benschop et al., 2015). Estos ítems (2, 8, 9 y 16) eran los mismos que fueron eliminados en las versiones cortas del DMQ-R (Kuntsche y Kuntsche, 2009; Mezquita et al., 2018).

Respecto de las fuentes de evidencia de la validez concurrente y predictiva, y de manera similar a lo hallado con el alcohol, aparentemente cada motivo de consumo de marihuana se relaciona de forma diferente con las variables de consumo de cannabis (Simons et al., 1998). Estudios trans- versales con el MMM (Simons et al., 1998) han encontrado que los motivos de animación se relacionan con el uso de cannabis (Buckner, 2013; Foster, Allan, Zvolensky, y Schmidt, 2014; Simons, Simons, y Spelman, 2016), y también con los problemas derivados del consumo de cannabis (Simons, Gaher, Correia, Hansen, y Christopher, 2005). Los motivos de afrontamiento se han relacionado con el consumo de cannabis, problemas derivados del consumo de cannabis (Buckner, 2013; Buckner y Zvolensky, 2014; Buckner, Zvolensky, y Schmidt, 2012; Foster et al., 2014; Simons et al., 2016) y dependencia de cannabis (Moitra, Christopher, Anderson, y Stein, 2015). Los motivos de expansión se han relacionado con la frecuencia de consumo de cannabis y la dependencia de cannabis en mujeres con síntomas de trastorno límite de la personalidad (Chabrol et al., 2005), y también con los problemas derivados del consumo en una muestra de consumidores actuales de cannabis (Buckner y Zvolensky, 2014). Por último, se ha hallado una relación negativa entre los motivos sociales y de conformidad con la frecuencia de consumo de cannabis (Buckner, 2013; Buckner y Zvolensky, 2014), y tan solo una relación positiva entre los motivos de conformidad y los problemas derivados del consumo de cannabis (Buckner et al., 2012; Foster et al., 2014).

Se han realizado muy pocos estudios prospectivos sobre la relación de los motivos de consumo de marihuana y el propio consumo de marihuana. Anderson, Sitney y White (2015) estudiaron una muestra comunitaria de 434 jóvenes y encontraron que los motivos de consumo de refuerzo positivo se asociaban con un aumento del consumo de cannabis y con los problemas derivados del consumo de cannabis, mientras que los motivos de consumo de refuerzo negativo fueron predictores de los problemas derivados del consumo de cannabis cuando se controlaron los motivos y el consumo previos. Los motivos de expansión en la adolescencia se han relacionado con un consumo de cannabis más bajo en la adultez temprana. Liebregts et al. (2013) encontraron que los motivos de afrontamiento eran predictores de la dependencia de la marihuana en una cohorte de consumidores frecuentes de cannabis.

El objetivo general de este estudio es desarrollar una versión corta del MMM que pudiera facilitar la inclusión de los motivos de consumo de cannabis en las sesiones de evaluación, o en programas de prevención o tratamiento, cuyos tiempos de administración y espacio son limitados (Kuntsche y Kuntsche, 2009). Nuestra intención fue desarrollar una versión corta que incluyese los ítems realmente apropiados para valorar los motivos de consumo de marihuana y eliminar otros ítems que habían demostrado no contribuir en la evaluación del constructo (Benschop et al., 2015). Los fines específicos de este estudio fueron: 1) traducir y adaptar el MMM al español; 2) crear una versión corta del instrumento usando análisis de los ítems y de Rasch; 3) explorar la estructura de la versión corta del cuestionario; y 4) estudiar distintas fuentes de evidencia 
de validez de criterio del cuestionario. Nuestra hipótesis era que, a pesar del menor número de ítems, el MMM SF mostraría una estructura, índices de fiabilidad y evidencia para predecir variables de consumo de cannabis al menos igual de buenas que el MMM original.

Tabla 1. Datos descriptivos de la muestra final de 232 participantes.

\begin{tabular}{|c|c|c|}
\hline Datos descriptivos & & $\begin{array}{l}\% \text { de la } \\
\text { muestra total }\end{array}$ \\
\hline \multirow[t]{3}{*}{ Nivel de estudios alcanzado } & Escuela primaria & 9,05 \\
\hline & Escuela secundaria & 53,02 \\
\hline & Universidad & 37,98 \\
\hline \multirow[t]{6}{*}{ Nivel de ingresos } & Menos de $€ 450$ & 55,17 \\
\hline & $€ 450-€ 900$ & 15,52 \\
\hline & $€ 900-€ 1500$ & 16,38 \\
\hline & $€ 1500-€ 2100$ & 8,19 \\
\hline & $€ 2100-€ 2700$ & 4,31 \\
\hline & $€ 2700-€ 3600$ & 0,43 \\
\hline \multirow[t]{7}{*}{ Renta familiar } & Menos de $€ 450$ & 14,36 \\
\hline & $€ 450-€ 900$ & 21,03 \\
\hline & $€ 900-€ 1500$ & 24,10 \\
\hline & $€ 1500-€ 2100$ & 21,54 \\
\hline & $€ 2100-€ 2700$ & 11,28 \\
\hline & $€ 2700-€ 3600$ & 5,13 \\
\hline & $€ 900-€ 1500$ & 2,56 \\
\hline \multirow[t]{4}{*}{ Convivencia (vive con) } & Solo/a & 9,48 \\
\hline & Padres o familia & 58,62 \\
\hline & Pareja & 20,26 \\
\hline & $\begin{array}{l}\text { Otros (p. ej., } \\
\text { compañeros/as de piso) }\end{array}$ & 11,64 \\
\hline \multirow[t]{3}{*}{ Ocupación principal } & Estudiante & 67,10 \\
\hline & Empleado/a & 28,57 \\
\hline & Desempleado/a & 4,33 \\
\hline
\end{tabular}

\section{Método}

\section{Participantes}

La muestra original estaba compuesta de 390 participantes. No obstante, de forma similar a los estudios previos sobre motivos, analizamos únicamente los datos de las personas que habían consumido marihuana al menos una vez en su vida. De los 236 evaluados, dos no completaron el MMM, y dos aparentemente contestaron los cuestionarios de manera aleatoria. La muestra final estaba compuesta de 232 participantes $\left(50,53 \%\right.$ hombres, $\left.e d a d_{\text {media }}=25,11, S D=7,58\right)$, con un intervalo de edades de entre 16-58 años. En la Tabla 1 se presentan los demás datos descriptivos de la muestra.

\section{Procedimiento}

Aplicamos las recomendaciones de Muñiz, Elosua y Hambleton (2013) para la traducción y adaptación de cuestionarios. Primero, tras revisar la literatura, escogimos el cuestionario más adecuado para evaluar los motivos de consumo de cannabis. Segundo, solicitamos los permisos para usar, traducir y adaptar el cuestionario. Para la traducción y adaptación del cuestionario, dos investigadores con experiencia en la construcción de tests psicométricos, y familiarizados con estudios sobre el cannabis, tradujeron los ítems al castellano. Después, un profesor de inglés, desconocedor del inventario, realizó su traducción inversa. El análisis de la traducción inversa indicó que la versión en castellano se podía considerar comparable a la escala original. También tuvimos en cuenta diferencias culturales entre España y EEUU, país donde se desarrolló el cuestionario original, cuando adaptamos el MMM.

Usamos dos métodos en el reclutamiento de la muestra. Primero, evaluamos a los participantes que estudiaban formación profesional en diferentes institutos de la provincia de Castellón (zona este de España): Politécnico, Matilde Salvador y Salvador Seguí $(N=149)$. Durante las sesiones de evaluación, psicólogos formados para este fin siguieron unas instrucciones estandarizadas: repartieron las escalas, garantizaron la confidencialidad, y animaron a los participantes a que respondieran con sinceridad. En este caso, la Consejería de Educación de la Generalitat Valenciana aprobó el uso de la batería de cuestionarios en la sesión de evaluación. Segundo, creamos una versión online de la batería de cuestionarios y los participantes la respondieron vía Internet $(N=83)$. Completaron las escalas en respuesta a un anuncio publicado en Facebook. En este caso, la información sobre el estudio, incluyendo las cuestiones deontológicas, fueron presentadas en la primera página de la batería, tras su aprobación por parte del Comité Ético de la Universitat Jaume I.

En ambos casos, todos los participantes facilitaron su consentimiento para participar en el estudio, cumplimentaron los cuestionarios de forma voluntaria y anónima, y no percibieron compensación alguna por su participación.

\section{Medidas}

El uso de marihuana se evaluó con la escala Cannabis and Other Drugs Intake Scale (CODIS), desarrollada por nuestro equipo de investigación a partir de otros instrumentos. El CODIS incluye una medida de la frecuencia de consumo de cannabis a lo largo de la vida ( $\mathrm{Fq}$ life: Indica si has consumido cannabis desde nunca 0 a diariamente 5), frecuencia de consumo de cannabis entre semana ( $\mathrm{Fq}$ entre semana: número de días en los cuales fumas cannabis entre lunes y jueves: 0 - 4), y en fin de semana (Fq fin de semana: número de días en los cuales fumas cannabis entre viernes y domingo: $O$ 3), número de porros fumados entre semana (Qn entre semana) y en fin de semana (Qn fin de semana), frecuencia de consumo durante el periodo de consumo de cannabis más intenso (Fq más intenso: durante tu periodo de consumo más intenso, con qué frecuencia fumabas, entre nunca 0 a varias 
veces por día 6) y número de porros fumados entre semana (Qn entre semana periodo más intenso) y en fin de semana (Qn fin de semana periodo más intenso) durante una semana típica del periodo de consumo de cannabis más intenso. Mientras que las preguntas sobre el número de porros fumados eran de respuesta abierta, las que preguntaban sobre la frecuencia tenían un formato de respuesta tipo Likert.

El MMM (Simons et al., 1998) está compuesto por 25 ítems, y cada ítem contribuye a una de las cinco subescalas: social, afrontamiento, animación, conformidad y expansión. Después de considerar todas las ocasiones durante las cuales los participantes habían fumado marihuana, indicaron la frecuencia con la cual fumaban por la razón especificada en cada ítem en una escala tipo Likert de 5 puntos, desde 1 (casi nunca/nunca) hasta 5 (casi siempre/siempre).

El cuestionario Cannabis Problems Questionnaire (CPQ; Copeland, Gilmour, Gates, y Swift, 2005) está compuesto por 27 ítems que evalúan los problemas derivados del consumo de cannabis. Los participantes informaron si habían experimentado una serie de consecuencias debidas a su consumo de marihuana durante los últimos 3 meses. Los ítems eran dicotómicos (sí/no). En esta muestra, el alfa de Cronbach de la escala fue 0,90 .

\section{Imputación de datos perdidos}

Los valores perdidos en el MMM en la muestra final $(N=$ 232) eran $0,21 \%$ de todos datos. Por esta razón, en el AFC y el análisis de los ítems y Rasch sustituimos el valor perdido de cada participante por la media de la escala a la que pertenecía el valor perdido (Bentler, 2006). En el análisis de regresión, realizamos el análisis de los datos disponibles (pair-wise), aunque había únicamente 19 valores ausentes en total en las medidas de consumo de cannabis y en los datos descriptivos.

\section{Análisis de datos}

Estrategias de selección de ítems. El objetivo era reducir la extensión de la escala original a la vez que evaluar de forma adecuada el constructo. Combinamos el análisis clásico de ítems y los procedimientos de medida de Rasch para seleccionar los ítems (Meyer, 2014). Usamos el Joint Maximum Likelihood Estimation (JMLE). Primero, calculamos las correlaciones entre el ítem y la escala total (i.e., discriminación de los ítems clásica). Al considerar el número de puntos en la escala de Likert, el índice de discriminación debería ser 0,60 o mayor. Segundo, evaluamos el persona-ítem outfit e infit usando los estadísticos Unweighted Mean Square (UMS) y Weighted Mean Square (WMS). Para ambos casos, se recomiendan valores entre 0,80 y 1,20 , y prestar mayor atención a valores más altos que a los más bajos (Meyer, 2014). Antes de implementar el análisis de ítems, se confirmaron las suposiciones respecto de dimensionalidad y de independencia local.
Además de estas consideraciones estadísticas, cuando los ítems mostraron buenos índices, seleccionamos los ítems que medían aspectos diferentes de la escala. Los ítems que eran un componente esencial de una escala de motivos no se eliminaron (véase Mezquita, Camacho, Suso, Ortet, y Ibáñez, 2018, para un procedimiento similar). Todos los ítems fueron analizados usando el software jMetrik (Meyer, 2014).

Evaluación de la estructura del cuestionario. Después de seleccionar el grupo de 15 ítems, y de manera similar a estudios anteriores realizados con el MMM (Zvolensky et al., 2007) y el DMQ-R SF (Kuntsche y Kuntsche, 2009; Mezquita et al., 2018), realizamos un AFC de cinco factores correlacionados. También analizamos otros posibles modelos hallados en la literatura con el DMQ-R (Cooper, 1994; Hauck-Filho, Pereira y Cooper, 2012): un modelo unidimensional en el cual todos los ítems cargaban en un factor único; un modelo de dos factores de refuerzo positivo (social, animación y expansión) y negativo (afrontamiento y conformidad); y un modelo de dos factores de fuente de refuerzo interna (animación, afrontamiento y expansión) y externa (social y conformidad).

Dada la distribución anormal de nuestros datos, usamos los métodos robustos de estimación de Satorra-Bentler para todos los análisis de modelos de ecuaciones estructurales. Para considerar que un modelo tiene un ajuste excelente, el ${ }_{\mathrm{s} \text { - }} \chi^{2}$ tiene que ser no significativo, pero esto no es común en el AFC. Por lo tanto, es interesante usar otros índices de ajuste para comparar modelos competidores: el índice de ajuste no normalizado (NNFI), el índice de ajuste comparativo (CFI), el índice de ajuste incremental (IFI), el índice de ajuste de McDonald (MFI), el error medio cuadrático de aproximación (RMSEA), y el criterio de información de Akaike (AIC). Valores más bajos de AIC indican mejor ajuste. Un modelo con NNFI, CFI, IFI, y MFI $\geq 0,90$, RMSEA $\leq 0,10$ se considera tener un ajuste aceptable, y NNFI, CFI, IFI y MFI $\geq 0,95$, y RMSEA $\leq 0,06$ un ajuste adecuado (Byrne, 2006).

Fiabilidad de puntuaciones. Para someter a prueba la fiabilidad de las subescalas, calculamos los alfas de Cronbach y omegas ordinales (Dunn, Baguley, y Brunsden, 2014) con IC 95\% usando los software jMetrik (Meyer, 2014) y R 3.4.0 (R Core Team, 2013), respectivamente.

Invarianza métrica entre grupos por sexo. Se realizaron Modelos de Ecuaciones Estructurales (MES) para determinar la invarianza métrica del cuestionario entre hombres y mujeres. En un paso inicial, sometimos a prueba el modelo de forma separada para cada grupo por sexo. Segundo, exploramos la invarianza configural entre grupos mediante un análisis multigrupo entre hombres y mujeres. Probamos las invarianza métrica, escalar y del error de medida (Milfont y Fischer, 2010). Evaluamos la bondad de ajuste relativa entre los modelos cada vez más restringidos usando el cálculo del test de diferencias del chi-cuadrado ${ }_{S-B} \mathrm{X}^{2}$ 
(Satorra y Bentler, 2001). Todos los AFC fueron realizados usando la versión 6.1 del software EQS (Bentler y Wu, 2002).

Relación entre motivos de consumo de marihuana y las variables de consumo de marihuana. Los análisis descriptivos, correlaciones de Pearson y análisis de regresión se hicieron con el paquete estadístico SPSS versión 22 (IBM Corp, 2013). Realizamos ocho análisis de regresión diferentes, controlando el efecto del sexo y la edad. En estos análisis, los motivos de consumo de marihuana fueron las variables independientes, y las variables de consumo de marihuana fueron las variables dependientes. Para los problemas relacionados con el consumo de cannabis, realizamos un análisis de regresión adicional controlando por la frecuencia y cantidad de consumo de cannabis.

Tabla 2. Análisis de los Ítems y de Rasch del Marijuana Motives Questionnaire.

\begin{tabular}{|c|c|c|c|c|c|}
\hline Subescala & Ítems & Discriminación & Dificultad & UMS & WMS \\
\hline \multirow[t]{5}{*}{ Social } & Item 3 & ,66 &,- 19 & 81 & 85 \\
\hline & Item 5 & ,42 & ,87 & 1,38 & 1,70 \\
\hline & Item 11 &, 65 &,- 20 & ,92 & 90 \\
\hline & Item 14 & ,74 &,- 02 & ,65 & ,70 \\
\hline & Item 16 &, 57 &,- 46 & 1,12 & 1,21 \\
\hline \multirow[t]{5}{*}{ Animación } & Item 7 & ,74 &,- 87 & ,73 & ,73 \\
\hline & Item 9 &, 34 & 1,49 & 2,23 & 2,23 \\
\hline & Item 10 & ,68 &,- 06 & ,98 & ,98 \\
\hline & Item 13 &, 74 &,- 68 & ,71 & ,71 \\
\hline & Item 18 & ,64 & ,13 & ,95 & ,95 \\
\hline \multirow[t]{5}{*}{ Afrontamiento } & Item 1 & ,80 &,- 14 & ,76 & ,86 \\
\hline & Item 4 & ,78 &,- 12 & ,94 & ,93 \\
\hline & Item 6 & ,72 &,- 32 & 1,07 & 1,11 \\
\hline & Item 15 & ,69 & ,87 & 1,41 & 1,37 \\
\hline & Item 17 &, 80 &,- 28 &, 87 & ,92 \\
\hline \multirow[t]{5}{*}{ Conformidad } & Item 2 &, 55 &,- 89 & 1,43 & 1,53 \\
\hline & Item 8 & ,66 & 1,06 & ,94 & 1,18 \\
\hline & Item 12 & ,79 &,- 45 & ,64 & ,75 \\
\hline & Item 19 &, 74 &,- 01 & ,98 & 1,05 \\
\hline & Item 20 & ,84 &, 28 &, 53 &, 58 \\
\hline \multirow[t]{5}{*}{ Expansión } & Item 21 &, 62 &, 60 & 1,13 & 1,37 \\
\hline & Item 22 &, 76 &,- 40 & ,98 & ,90 \\
\hline & Item 23 & ,83 &,- 18 & ,63 & ,69 \\
\hline & Item 24 & ,68 & ,46 & 91 & 1,11 \\
\hline & Item 25 & ,69 &,- 48 & 1,09 & 1,05 \\
\hline
\end{tabular}

Nota. Los ítems incluidos en el MMM SF aparecen en negrita. El contenido de los ítems puede consultarse en Simons et al. (1998).

\section{Resultados}

\section{Selección de ítems}

En la Tabla 2 se muestran los resultados de los análisis de los ítems y de Rasch. Primero, considerando los índices de discriminación, eliminamos los ítems 5 y 16 de la escala de motivos sociales, el ítem 9 de la escala de animación, y el ítem 2 de la escala de conformidad. Segundo, considerando los índices de UMS y WMS, también eliminamos el ítem 15 de la escala de afrontamiento y el ítem 21 de la escala de expansión. De los ítems restantes, elegimos los que eran esenciales para la escala de motivos y los que tenían menor solapamiento de contenido (p. ej., "Porque me gusta la sensación" se mantuvo en lugar de "Porque me da una sensación placentera”). En la Tabla 2 se presenta en negrita el grupo final de 15 ítems.

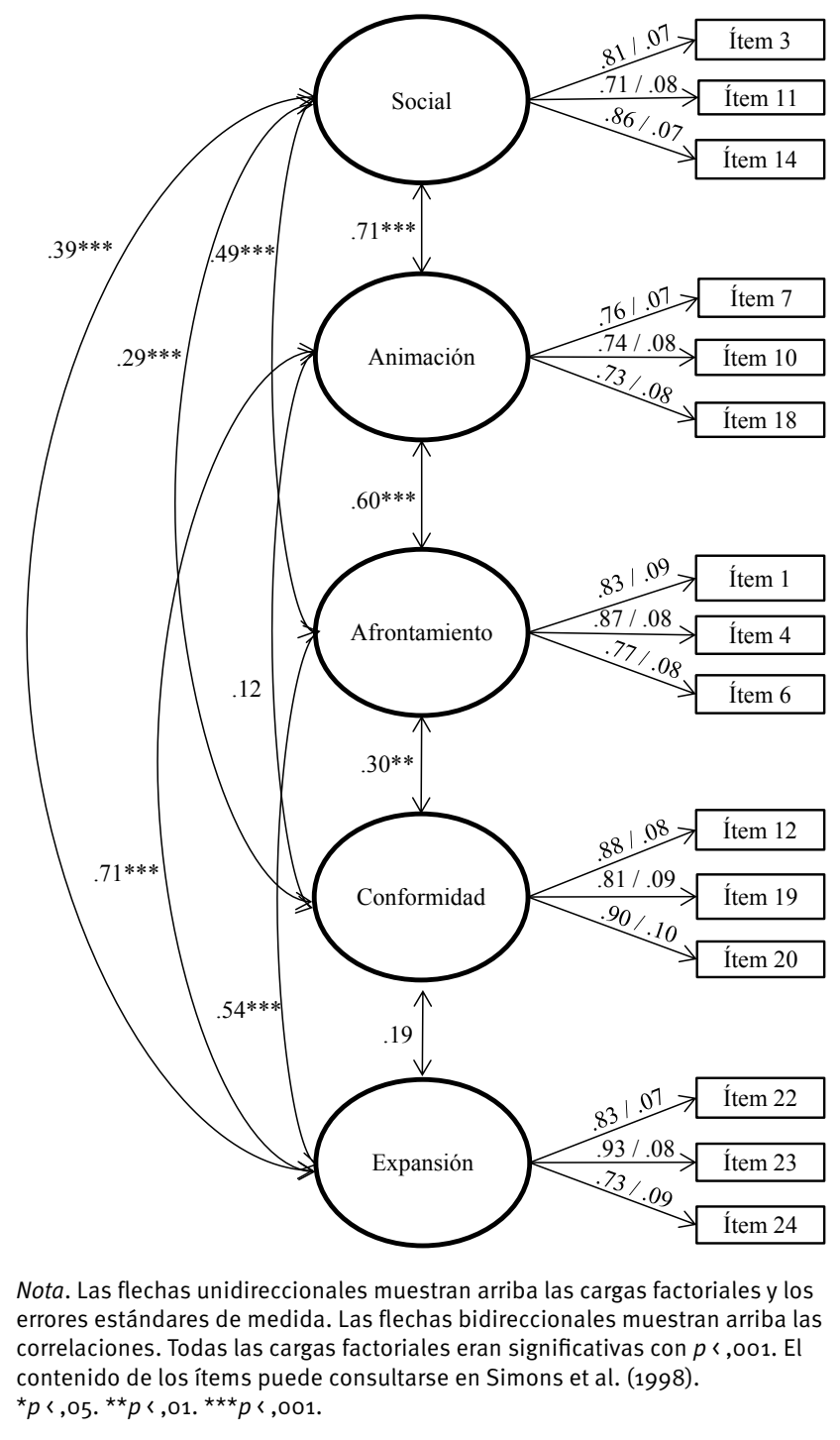

Figura 1. AFC correlacionado de la solución final de 15 ítems del MMM SF. 
Tabla 3. Índices de Ajuste de los Distintos Modelos Estructurales y Análisis Multi-Grupo entre Hombres y Mujeres del MMM SF.

\begin{tabular}{|c|c|c|c|c|c|c|c|c|c|c|c|}
\hline & & & S-BX $\mathbf{X}^{2}$ & g.l. & $p$ & NNFI & CFI & IFI & MFI & RMSEA $(90 \% \mathrm{Cl})$ & AIC \\
\hline MMM & $\begin{array}{l}\text { Modelo de cinco factores } \\
\text { correlacionados }\end{array}$ & Muestra íntegra & 497,51 & 265 &, 000 & ,837 & ,856 & 859 & ,606 &, $062(, 053,, 070)$ & $-32,49$ \\
\hline \multirow[t]{10}{*}{ MMM SF } & $\begin{array}{l}\text { Modelo de cinco factores } \\
\text { correlacionados }\end{array}$ & Muestra íntegra & 121,30 & 80 &, 002 & ,944 & ,958 & ,959 & ,915 & ,047 (,029, ,063) & $-38,70$ \\
\hline & Modelo unidimensional & Muestra íntegra & 549,56 & 90 &, 000 &, 450 &, 528 &, 535 & ,371 &, $149(, 136,, 160)$ & 369,56 \\
\hline & $\begin{array}{l}\text { Modelo de dos factores, } \\
\text { fuente interna vs. externa }\end{array}$ & Muestra íntegra & 509,42 & 89 &, 000 & ,491 &, 568 &, 575 & ,404 &, $143(, 131,, 155)$ & 331,42 \\
\hline & $\begin{array}{l}\text { Modelo de dos factores, } \\
\text { refuerzo positivo vs. } \\
\text { negativo }\end{array}$ & Muestra íntegra & 569,69 & 89 &, 000 &, 418 &, 507 &, 515 & ,355 &, $153(, 141,, 165)$ & 391,69 \\
\hline & $\begin{array}{l}\text { Modelo de cinco factores } \\
\text { correlacionados }\end{array}$ & Hombres & 119,13 & 80 & ,003 & ,896 & ,921 & ,925 & ,846 &, $065(, 038,, 088)$ & $-40,87$ \\
\hline & & Mujeres & 112,13 & 80 & 010 & ,900 & ,924 & ,923 & 870 & ,059 (,030, ,083) & $-47,87$ \\
\hline & $\begin{array}{l}\text { Análisis multigrupo del } \\
\text { modelo de cinco factores } \\
\text { correlacionados }\end{array}$ & Invarianza configural & 230,01 & 160 &, 000 & ,900 & ,924 & ,927 & ,860 &, $062(, 043,, 078)$ & $-89,99$ \\
\hline & & Invarianza métrica & 239,03 & 175 &, 001 & ,916 & ,930 & ,933 & ,871 &, $056(, 037,, 073)$ & $-110,97$ \\
\hline & & Invarianza escalar & 261,06 & 190 &, 000 & ,903 & ,925 & ,929 & ,855 &, $057(, 038,, 073)$ & $-118,94$ \\
\hline & & $\begin{array}{l}\text { invarianza del error } \\
\text { de medida }\end{array}$ & 259,24 & 205 & ,006 & ,929 & ,944 & ,946 & ,994 &, $051(, 031,, 068)$ & $-150,76$ \\
\hline
\end{tabular}

Tabla 4. Análisis descriptivos para la muestra íntegra y diferenciando entre hombres y mujeres.

\begin{tabular}{|c|c|c|c|c|c|c|c|c|c|c|}
\hline & \multicolumn{4}{|c|}{ Muestra íntegra } & \multicolumn{2}{|c|}{ Hombres } & \multicolumn{2}{|c|}{ Mujeres } & \multirow[b]{2}{*}{$t$} & \multirow[b]{2}{*}{$d$} \\
\hline & $\mathbf{x}$ & SD & $a$ & $\Omega$ & $\mathbf{x}$ & SD & $\mathbf{x}$ & SD & & \\
\hline Social & 5,75 & 2,95 &, $83(, 79,, 88)$ &, $82(, 79,, 86)$ & 6,03 & 3,19 & 5,47 & 2,68 & 1,44 & ,19 \\
\hline Animación & 7,09 & 3,41 &, $79(, 74,, 84)$ &, $79(, 73,, 83)$ & 7,60 & 3,80 & 6,57 & 2,88 & $2,33^{*}$ & ,31 \\
\hline Afrontamiento & 5,05 & 2,84 &, $86(, 82,, 91)$ &, $86(, 83,, 89)$ & 5,23 & 3,17 & 4,87 & 2,45 & ,97 & ,13 \\
\hline Conformidad & 3,68 & 1,88 & ,90 $(, 84,, 96)$ & ,89 (,86, ,91) & 3,52 & 1,64 & 3,83 & 2,10 & $-1,27$ &,- 16 \\
\hline Expansión & 4,65 & 2,53 & ,83 (,76, ,91) & ,86 (,83, ,89) & 4,86 & 2,66 & 4,43 & 2,38 & 1,32 & ,17 \\
\hline Fq entre semana & ,67 & 1,30 & & - & ,90 & 1,44 &, 45 & 1,10 & $2,66^{\star \star}$ & ,35 \\
\hline Fq fin de semana & ,79 & 1,12 & & - & ,90 & 1,18 & ,68 & 1,03 & 1,46 & ,20 \\
\hline Qn entre semana & 1,29 & 3,27 & & - & 1,67 & 3,70 & ,89 & 2,72 & 1,80 & ,24 \\
\hline Qn fin de semana & 1,65 & 3,16 & & - & 2,09 & 3,72 & 1,21 & 2,37 & $2,12^{\star}$ & ,28 \\
\hline $\begin{array}{l}\text { Fq periodo más } \\
\text { intenso }\end{array}$ & 3,33 & 1,99 & & - & 3,54 & 2,12 & 3,11 & 1,83 & 1,63 & ,22 \\
\hline $\begin{array}{l}\text { Qn periodo más } \\
\text { intenso entre } \\
\text { semana }\end{array}$ & 4,14 & 6,74 & & & 5,50 & 7,90 & 2,76 & 4,98 & $3,13^{\star \star}$ & ,41 \\
\hline $\begin{array}{l}\text { Qn periodo más } \\
\text { intenso } \\
\text { fin de semana }\end{array}$ & 4,77 & 6,19 & & & 5,82 & 7,05 & 3,70 & 4,99 & $2,63^{\star *}$ &, 35 \\
\hline $\begin{array}{l}\text { Problemas } \\
\text { relacionados con } \\
\text { el consumo de } \\
\text { cannabis }\end{array}$ & 2,51 & 3,99 & & & 3,26 & 4,66 & 1,75 & 3,01 & $2,92^{\star \star}$ & ,38 \\
\hline
\end{tabular}

Nota. Fq entre semana = frecuencia de consumo de cannabis entre semana; Fq fin de semana = frecuencia de consumo de cannabis en fin de semana; Qn entre semana = número de porros fumados entre semana; Qn fin de semana = número de porros fumados en fin de semana; Fq más intenso = frecuencia de consumo durante el periodo de consumo de cannabis más intenso; Qn más intenso entre semana = número de porros fumados durante una semana típica del periodo de consumo de cannabis más intenso; Qn más intenso fin de semana = número de porros fumados en un fin de semana durante una semana típica del periodo de consumo de cannabis más intenso. Alfas de Cronbach y coeficientes omega ordinales con $95 \%$ IC. Los valores del $d$ de Cohen de o,20, o,50, y o,80 corresponden a los tamaños de efecto pequeño, medio y grande, respectivamente (Cohen, 1992).

${ }^{\star} p<, 05 .{ }^{* \star} p<, 01 .{ }^{* \star *} p<, 001$ 
Tabla 5. Análisis de correlación de Pearson entre los motivos de consumo de cannabis y las variables de consumo de cannabis.

\begin{tabular}{|c|c|c|c|c|c|c|c|c|c|c|c|c|c|}
\hline & Social & Animación & Afrontamiento & Conformidad & Expansión & $\begin{array}{l}\text { Fq entre } \\
\text { semana }\end{array}$ & $\begin{array}{l}\text { Fq fin de } \\
\text { semana }\end{array}$ & $\begin{array}{l}\text { Qn entre } \\
\text { semana }\end{array}$ & $\begin{array}{l}\text { Qn fin de } \\
\text { semana }\end{array}$ & $\begin{array}{c}\text { Fq periodo } \\
\text { más } \\
\text { intenso }\end{array}$ & $\begin{array}{c}\text { Qn periodo } \\
\text { más intenso } \\
\text { entre semana }\end{array}$ & $\begin{array}{c}\text { Qn periodo } \\
\text { más intenso } \\
\text { fin de semana }\end{array}$ & $\begin{array}{l}\text { Problemas } \\
\text { derivados del } \\
\text { consumo de } \\
\text { cannabis }\end{array}$ \\
\hline Social & - & $.59^{\star \star \star}$ & $.44^{\star \star \star}$ & $.30^{\star \star \star \star}$ & $.38^{\star \star \star}$ & .12 & $.20 \star \star$ & $.14^{\star}$ & $.17^{\star \star}$ & $.24^{\star \star \star}$ & .12 & $.16^{*}$ & $.21^{\star \star}$ \\
\hline Animación & & - & $.52^{\star \star \star}$ & .10 & $.62^{\star \star \star}$ & $.35^{\star \star \star}$ & $.39^{\star \star \star}$ & $.31^{\star \star \star}$ & $.40^{\star \star \star}$ & $.51^{\star \star \star}$ & $.33^{\star \star \star}$ & $.36^{\star \star \star}$ & $.41^{\star \star \star}$ \\
\hline Afrontamiento & & & - & $.27^{\star \star \star}$ & $.50^{\star \star \star}$ & $.31^{\star \star \star}$ & $.38^{\star \star \star}$ & $.31^{\star \star \star}$ & $.35^{\star \star \star}$ & $.36^{\star \star \star}$ & $.25^{\star \star \star}$ & $.20^{\star \star}$ & $.43^{\star \star \star}$ \\
\hline Conformidad & & & & - & $.17^{\star}$ & -.12 & $-.17^{\star}$ & -.08 & -.13 & -.13 & -.10 & -.10 & -.12 \\
\hline Expansión & & & & & - & $.26^{\star \star \star}$ & $.30^{\star \star \star}$ & $.24^{\star \star \star}$ & $.28^{\star \star \star}$ & $.30^{\star \star \star}$ & $.17^{\star \star}$ & $.14^{\star}$ & $.36^{\star \star \star}$ \\
\hline
\end{tabular}

Nota. Fq entre semana = frecuencia de consumo de cannabis entre semana; Fq fin de semana = frecuencia de consumo de cannabis en fin de semana; Qn entre semana = número de porros fumados entre semana; Qn fin de semana = número de porros fumados en fin de semana; Fq más intenso = frecuencia de consumo durante el periodo de consumo de cannabis más intenso; Qn más intenso entre semana = número de porros fumados durante una semana típica del periodo de consumo de cannabis más intenso; Qn más intenso fin de semana = número de porros fumados en un fin de semana durante una semana típica del periodo de consumo de cannabis más intenso.

${ }^{*} p<, 05 .{ }^{* *} p<, 01 .{ }^{* *} p<, 001$.

Tabla 6. Análisis de regresión: Motivos de consumo de cannabis como predictores de las variables de consumo de cannabis.

\begin{tabular}{|c|c|c|c|c|c|c|c|c|c|c|c|c|c|c|c|c|c|c|c|}
\hline \multirow[t]{2}{*}{ VI } & \multicolumn{2}{|c|}{$\begin{array}{l}\text { Fq entre } \\
\text { semana }\end{array}$} & \multicolumn{2}{|c|}{$\begin{array}{l}\text { Fq fin de } \\
\text { semana }\end{array}$} & \multicolumn{2}{|c|}{$\begin{array}{l}\text { Qn entre } \\
\text { semana }\end{array}$} & \multicolumn{2}{|c|}{$\begin{array}{l}\text { Qn fin de } \\
\text { semana }\end{array}$} & \multicolumn{2}{|c|}{$\begin{array}{l}\text { Fq periodo } \\
\text { más intenso }\end{array}$} & \multicolumn{2}{|c|}{$\begin{array}{l}\text { Qn periodo } \\
\text { más intenso } \\
\text { entre } \\
\text { semana }\end{array}$} & \multicolumn{2}{|c|}{$\begin{array}{l}\text { Qn periodo } \\
\text { más intenso } \\
\text { fin de } \\
\text { semana }\end{array}$} & \multicolumn{2}{|c|}{$\begin{array}{l}\text { Problemas } \\
\text { derivados del } \\
\text { consumo de } \\
\text { cannabis }\end{array}$} & \multirow[t]{2}{*}{ VI } & \multicolumn{2}{|c|}{$\begin{array}{l}\text { Problemas } \\
\text { derivados del } \\
\text { consumo de } \\
\text { cannabis }\end{array}$} \\
\hline & $\beta$ & $\Delta \mathrm{R}^{2}$ & $\beta$ & $\Delta \mathrm{R}^{2}$ & $\beta$ & $\Delta \mathrm{R}^{2}$ & $\beta$ & $\Delta \mathrm{R}^{2}$ & $\beta$ & $\Delta R^{2}$ & $\beta$ & $\Delta \mathrm{R}^{2}$ & $\beta$ & $\Delta \mathrm{R}^{2}$ & $\beta$ & $\Delta R^{2}$ & & $\beta$ & $\Delta \mathrm{R}^{2}$ \\
\hline \multicolumn{20}{|l|}{ Paso 1} \\
\hline Sexo &,$- 18^{\star \star}$ &, $03^{*}$ &,- 10 & ,01 &,- 12 & ,02 &,$- 14^{\star}$ & ,02 &,- 11 & ,01 &,$- 21^{\star \star}$ &, $04^{\star \star}$ &,$- 17^{\star}$ &, $03^{*}$ &,$- 19^{\star \star}$ &, $04^{\star}$ & Sex &,- 09 &, $48^{\star \star \star}$ \\
\hline \multirow[t]{3}{*}{ Edad } & ,06 & &,- 01 & & ,03 & &,- 06 & &,- 02 & & ,02 & &,- 01 & &,- 01 & & Age &,- 01 & \\
\hline & & & & & & & & & & & & & & & & & Frequency &, $39^{\star \star \star}$ & \\
\hline & & & & & & & & & & & & & & & & & Quantity &, $32^{\star \star \star}$ & \\
\hline
\end{tabular}

Paso 2

\begin{tabular}{|c|c|c|c|c|c|c|c|c|c|c|c|}
\hline Social & $-14,19 * \star *$ &, $27^{\star \star \star}$ & $-08,15^{\star \star \star}$ & $-, 08,22^{\star \star \star}$ & $-, 07,31^{\star \star *}$ & $-11,14^{\star \star \star}$ & $-, 06,15^{\star \star \star}$ & $-, 05,28^{\star \star \star}$ & Social &,- 00 &, $05^{\star \star \star}$ \\
\hline Animación &, $29 * \star$ &, $23 * \star$ &, $22^{\star}$ &, $31^{\star \star *}$ &, $49^{\star \star \star}$ &, $34^{\star \star \star}$ &, $43^{\star \star *}$ &, $21^{\star}$ & Enhancement & ,05 & \\
\hline Afrontamiento &, $26 * \star \star$ &, $32^{\star \star \star}$ &, $26^{\star \star}$ &, $28^{\star \star \star}$ &, $23^{\star \star}$ &, $19^{\star}$ & ,10 &, $35^{\star \star \star}$ & Coping &, $18^{\star \star}$ & \\
\hline Conformidad &,$- 20^{\star \star}$ &,$- 30 \star \star \star$ &,$- 16^{\star}$ & 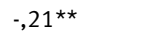 &,$- 22^{\star \star \star}$ &,$- 15^{\star}$ &,$- 13^{\star}$ &,$- 24^{\star \star \star}$ & Conformity &,$- 11^{\star}$ & \\
\hline Expansión & ,03 & ,06 & ,03 & ,01 &,- 06 &,- 07 &,- 13 & ,11 & Expansion & ,09 & \\
\hline
\end{tabular}

Nota. Fq entre semana = frecuencia de consumo de cannabis entre semana; Fq fin de semana = frecuencia de consumo de cannabis en fin de semana; Qn entre semana = número de porros fumados entre semana; Qn fin de semana = número de porros fumados en fin de semana; Fq más intenso = frecuencia de consumo durante el periodo de consumo de cannabis más intenso; Qn más intenso entre semana = número de porros fumados durante una semana típica del periodo de consumo de cannabis más intenso; Qn más intenso fin de semana = número de porros fumados en un fin de semana durante una semana típica del periodo de consumo de cannabis más intenso.

${ }^{*} p<, 05 .{ }^{* \star} p<, 01 .{ }^{* * *} p<, 001$.

\section{Fuentes de evidencia de validez de la estructura de MMM}

En la Tabla 3 se presentan los índices de ajuste del modelo de cinco factores correlacionados de las escalas MMM y MMM SF. Mientras que los índices de ajuste del MMM no fueron aceptables, los del MMM SF fueron, en general, adecuados. El modelo de cinco factores correlacionados del MMM SF también mostró mejores índices de ajuste que el modelo unidimensional y el de dos factores (Tabla 3). En la Figura 1 se muestran las cargas factoriales, errores estándares y covarianzas del modelo de cinco factores del MMM SF.

\section{Invarianza métrica de la escala entre grupos de sexo}

Cuando dividimos la muestra en hombres y mujeres, los índices de ajuste del MMM SF fueron ligeramente peores que para la muestra íntegra, aunque aceptables (Tabla 3). Por lo tanto, exploramos la invarianza configural entre grupos, y los índices de ajuste fueron aceptables (Tabla 3). Al añadir restricciones a las cargas factoriales $\left({ }_{\mathrm{s}-\mathrm{B}} \mathrm{X}^{2}{ }_{\mathrm{dif}}(15)=13,61, p=, 56\right)$, las medias $\left({ }_{\mathrm{s}-\mathrm{B}} \mathrm{X}_{\text {dif }}(15)=23,15, p=, 08\right)$ y los errores de medida $\left({ }_{\mathrm{s} B \mathrm{~B}} \mathrm{X}_{\text {dif }}(15)=8,65, p=, 90\right)$ entre hombres y mujeres los modelos no mostraron disminuciones significativas de ajuste. Esto indica que el MMM SF mostró invarianza métrica, escalar, y de los errores de medida entre grupos por sexo. 


\section{Fiabilidad de puntuaciones}

En la Tabla 4 se presentan los coeficientes alfas de Cronbach y omegas ordinales de las escalas con IC 95\%. La fiabilidad de todas las escalas varió entre buena y excelente (con todos los coeficientes alfas y omegas ordinales entre $0,79-0,90)$.

\section{Fuentes de evidencia de validez: motivos como predictores de diferentes variables de consumo de marihuana}

En la Tabla 4 se presentan los análisis descriptivos del MMM SF y las variables de consumo de marihuana. Los hombres obtuvieron puntuaciones significativamente más altas en los motivos de animación, frecuencia de consumo entre semana, cantidad de consumo en fin de semana, cantidad de consumo de marihuana durante el periodo de consumo más intenso entre semana y en fin de semana, y problemas derivados del consumo de cannabis. No obstante, el efecto de las diferencias fue mínima. El análisis correlacional mostró que las correlaciones más fuertes se hallaban entre los motivos internos de consumo de marihuana (animación, afrontamiento y expansión) y las variables de consumo de marihuana (Tabla 5).

El análisis de regresión mostró que los motivos de animación, afrontamiento y baja conformidad eran predictores de la frecuencia y la cantidad de marihuana fumada entre semana y en fin de semana (Tabla 6). El mejor predictor de la frecuencia y la cantidad de consumo durante el periodo de consumo más intenso fueron los motivos de animación, que tuvieron una asociación más fuerte con el consumo de fin de semana que con el de entre semana. A pesar de que los motivos de afrontamiento, animación y baja conformidad eran predictores de problemas relacionados con el consumo de cannabis, el efecto de los motivos de animación no era significativo cuando se controlaba el efecto de frecuencia y cantidad de consumo (Table 6).

\section{Discusión}

Los objetivos de este estudio fueron traducir y adaptar el MMM SF al castellano, explorar su estructura factorial y fiabilidad, y evaluar diferentes fuentes de validez de criterio (predictores de variables de consumo de marihuana). Los análisis de los ítem y de Rasch aportaron un grupo final de 15 ítems, tres por escala, con cargas factoriales significativas (todas fueron de 0,71 o superior). Los doce ítems de las escalas social, animación, afrontamiento y conformidad eran los mismos que los anteriormente retenidos en la versión corta del DMQ-R (Kuntsche y Kuntsche, 2009; Mezquita et al., 2018). Esto es importante para futuros estudios comparativos sobre motivos de consumo de sustancias. Si se encuentran diferencias entre motivos de consumo de drogas, éstas no se deben atribuir a las diferencias entre los cuestionarios, dada la equivalencia entre el MMM SF y el DMQ-R SF. La solución final de la escala de motivos de expansión de 3 ítems estaba compuesta por los tres ítems con las cargas factoriales más altas en la validación del cuestionario original (Simons et al., 1998). El cuestionario también mostró invarianza métrica entre hombres y mujeres. Como resultado, el MMM SF es un instrumento apropiado para comparar los motivos de consumo de marihuana por sexo.

Respecto de la fiabilidad de las puntuaciones, todas los alfas de Cronbach fueron superiores al punto de corte estándar de 0,70, a pesar de que escalas cortas suelen tener consistencias internas más bajas que escalas más largas. Cuando estudios previos exploraron los motivos de consumo de marihuana, los motivos de animación fueron los más informados, seguidos por los motivos sociales, de expansión, de afrontamiento y de conformidad (Buckner et al., 2012; Foster et al., 2014; Simons et al., 2016, 1998; Zvolensky et al., 2007). No obstante, en este estudio, los participantes informaron más motivos de afrontamiento que de expansión. Esto no fue debido a la brevedad del cuestionario, pero es indicativo de la existencia de algunas diferencias culturales que podrían ser objeto de análisis en estudios transculturales futuros.

Cuando exploramos las intercorrelaciones entre las escalas de motivos, las correlaciones más fuertes se hallaban entre los motivos de animación y de expansión. Este resultado era de esperar porque, como ocurre con los motivos de animación, en los motivos de expansión el consumo actúa como un refuerzo positivo cuya fuente de refuerzo es interna. Las correlaciones más bajas se observaron entre los motivos de conformidad y las demás escalas, como ocurrió en estudios anteriores (Simons et al., 1998; Zvolensky et al., 2007).

Respecto de las fuentes de validez de criterio, ambos motivos de afrontamiento y de animación estaban asociados de forma similar con la frecuencia y la cantidad fumada entre semana y en fin de semana. Este resultado fue diferente al hallado para alcohol, dado que estudios anteriores han mostrado que los motivos de animación están principalmente relacionados con el consumo en fin de semana, mientras que los motivos de afrontamiento están asociados con el consumo de alcohol entre semana (Mezquita, Ibáñez, Moya, Villa, y Ortet, 2014; Studer et al., 2014). De todas maneras, se hallaron algunas similitudes entre las sustancias. Los motivos de animación fueron el mejor predictor no solo de la frecuencia de consumo durante el periodo de consumo más intenso, sino también de la cantidad de consumo entre semana y en fin de semana durante el periodo de consumo más intenso. Estudios anteriores sobre alcohol también han informado que los motivos de animación son los mejores predictores de consumo de alcohol alto y del binge drinking (Cooper, 1994; McCabe, 2002). Por último, el hecho de que la asociación entre los motivos de animación y los problemas derivados del consumo de cannabis desapareciera cuando se controlaba por el efecto de la frecuencia y la cantidad de consumo de can- 
nabis, sugiere que la asociación estaba mediada por el uso de marihuana (Simons et al., 2005). En línea con esta idea, resultados anteriores sobre motivos y problemas derivados del consumo de alcohol han obtenido hallazgos similares (Mezquita et al., 2014). Es más, según estudios anteriores sobre marihuana (Simons et al., 2005) y alcohol (Mezquita et al., 2018, 2014), el motivo de afrontamiento es el mejor predictor de los problemas derivados del consumo de sustancias, incluso cuando se controla por las sustancias, sugiriendo que este motivo es un factor de vulnerabilidad para trastornos relacionados con el consumo de marihuana.

El análisis de regresión encontró una asociación negativa entre los motivos de conformidad y todas las variables de consumo de marihuana (Buckner y Zvolensky, 2014; Simons et al., 1998; Zvolensky et al., 2007). Otros estudios anteriores que incluyeron los motivos del consumo de alcohol hallaron resultados similares y ofrecieron interpretaciones diferentes de estos hallazgos (p. ej., Grant, Stewart, O'Connor, Blackwell, y Conrod, 2007; Mezquita, Stewart, Kuntsche y Grant, 2016; Kuntsche, Wiers, Janssen, y Gmel, 2010). Por un lado, este resultado podría deberse a un efecto de supresión (Grant et al., 2007). La definición clásica del efecto de supresión en un análisis de regresión es que una covariable potencial no relacionada con la variable de resultado (p. ej., tiene una correlación bivariada de cero) aumenta el ajuste del modelo general en la regresión cuando se añade dicha covariable (p. ej., motivos de conformidad) al modelo (Tu, Gunnell, y Gilthorpe, 2008). No obstante, esta explicación es improbable porque incluso cuando algunas de las correlaciones entre motivos de conformidad y las variables de consumo de marihuana fueron no significativas, mostraron una asociación negativa, con una tendencia hacia la significanción en la mayoría de los casos. Por otro lado, es lógico que las personas que informan motivos de conformidad indiquen una frecuencia baja de consumo de marihuana y que fumen cantidades pequeñas de marihuana. Con el fin de sentirse parte de un grupo, pueden ser suficientes unas pocas inhalaciones para lograr dicho fin; i.e., estar colocado puede ser incluso contraproducente respecto del objetivo de no sentirse excluidos (Kuntsche et al., 2010).

Para concluir, los motivos de expansión no estaban asociados de forma significativa con las variables de consumo de marihuana en los análisis de regresión. Estos resultados podrían deberse a las correlaciones altas encontradas entre los motivos de expansión y de animación, pero también a la asociación de los mismos con los motivos de afrontamiento (Figura 1 y Tabla 5). Las intercorrelaciones altas entre las escalas de motivos pueden disipar la influencia de los motivos de expansión sobre las variables de consumo de marihuana (Studer et al., 2014).

Este estudio no está exento de limitaciones. Primero, el tamaño de la muestra es modesto, igual que en estudios anteriores (Simons et al., 1998; Zvolensky et al., 2007). En parte se debe al hecho de que el cuestionario fue diseñado para ser usado con participantes que habían consumido cannabis al menos una vez en su vida, y que el consumo de cannabis no es tan frecuente como el consumo de alcohol o tabaco. No obstante, si consideramos que el cuestionario tiene únicamente 15 ítems, entonces podríamos considerar el tamaño de la muestra como adecuada. Segundo, debido al diseño transversal del estudio, no fue posible explorar la fiabilidad test-retest de las puntuaciones y las fuentes de validez de las escalas de motivos para predecir las variables de consumo de marihuana. Tercero, los motivos de consumo de cannabis no son las únicas variables proximales al consumo de cannabis a tener en cuenta (Lloret Irles, Morell-Gomis, Laguía, y Moriano, 2018). Han de estudiarse otras variables para obtener una visión completa del uso de cannabis en consumidores. Por último, el uso de cannabis podría evaluarse con mayor objetividad en lugar de exclusivamente mediante autoinformes (Casajuana et al., 2017).

En resumen, los resultados apoyan la idea de que el MMM SF es mejor (en términos de validez estructural), o al menos igual de bueno (en términos de la fiabilidad de sus puntuaciones y capacidad para predecir las variables de consumo de marihuana), que el MMM. El cuestionario parece ser una herramienta útil para valorar los motivos de consumo de marihuana cuando el tiempo del administrador es limitado, especialmente cuando se aplican varios instrumentos de evaluación.

\section{Reconocimientos}

La financiación para este estudio fue aportada en parte por los proyectos de investigación PSI2015-67766-R del Ministerio de Economía y Competitividad de España, y GV/2016/158 de la Generalitat Valenciana.

\section{Conflicto de intereses}

Lo autores declaran la inexistencia de conflicto de intereses.

\section{Referencias}

Anderson, K. G., Sitney, M. y White, H. R. (2015). Marijuana motivations across adolescence: Impacts on use and consequences. Substance Use $\mathcal{E}$ Misuse, 50, 292-301. doi: 10.3109/10826084.2014.977396.

Benschop, A., Liebregts, N., van der Pol, P., Schaap, R., Buisman, R., van Laar, M., ... Korf, D. J. (2015). Reliability and validity of the Marijuana Motives Measure among young adult frequent cannabis users and associations with cannabis dependence. Addictive Behaviors, 40, 91-95. doi:10.1016/j.addbeh.2014.09.003.

Bentler, P. M. (2006). EQS 6 structural equations program manual. Encino, CA: Multivariate Software. 
Bentler, P. M. y Wu, E. J. C. (2002). EQS 6 for Windows User's Guide [Computer software]. Software, Inc.

Buckner, J. D. (2013). College cannabis use: The unique role of social norms, motives, and expectancies. Journal of Studies on Alcohol and Drugs, 74, 720-726. doi:10.15288/ jsad.2013.74.720.

Buckner, J. D. y Zvolensky, M. J. (2014). Cannabis and related impairment: The unique roles of cannabis use to cope with social anxiety and social avoidance. The American Journal on Addictions, 23, 598-603. doi:10.1111/ j.1521-0391.2014.12150.x.

Buckner, J. D., Zvolensky, M. J. y Schmidt, N. B. (2012). Cannabis-related impairment and social anxiety: The roles of gender and cannabis use motives. Addictive Behaviors, 37, 1294-1297. doi:10.1016/j.addbeh.2012.06.013.

Byrne, B. M. (2006). Structural equation modeling with EQS: Basic concepts, applications, and programming, 2nd ed. Mahwah, NJ: Erlbaum.

Casajuana, C., López-Pelayo, H., Balcells, M. M., Miquel, L., Teixidó, L.,Colom, J. y Gual, A. (2018). Working on a Standard Joint Unit: A pilot test. Adicciones, 29, 227-232. doi:10.20882/adicciones.721.

Chabrol, H., Ducongé, E., Casas, C., Roura, C. y Carey, K. B. (2005). Relations between cannabis use and dependence, motives for cannabis use and anxious, depressive and borderline symptomatology. Addictive Behaviors, 30, 829-840. doi:10.1016/j.addbeh.2004.08.027.

Cooper, M. L. (1994). Motivations for alcohol use among adolescents: Development and validation of a four-factor model. Psychological Assessment, 6, 117-128. doi:10.1037/1040-3590.6.2.117.

Copeland, J., Gilmour, S., Gates, P. y Swift, W. (2005). The Cannabis Problems Questionnaire: Factor structure, reliability, and validity. Drug and Alcohol Dependence, 80, 313-319. doi:10.1016/j.drugalcdep.2005.04.009.

Dunn, T. J., Baguley, T. y Brunsden, V. (2014). From alpha to omega: A practical solution to the pervasive problem of internal consistency estimation. British Journal of Psychology, 105, 399-412. doi:10.1111/bjop.12046

Foster, D. W., Allan, N. P., Zvolensky, M. J. y Schmidt, N. B. (2014). The influence of cannabis motives on alcohol, cannabis, and tobacco use among treatment-seeking cigarette smokers. Drug and Alcohol Dependence, 146, 81-88. doi:10.1016/j.drugalcdep.2014.11.013.

Grant, V. V, Stewart, S. H., O'Connor, R. M., Blackwell, E. y Conrod, P. J. (2007). Psychometric evaluation of the five-factor Modified Drinking Motives Questionnaire-Revised in undergraduates. Addictive Behaviors, 32, 2611-2632.

Hauck-Filho, N., Teixeira, M. A. P. y Cooper, M. L. (2012). Confirmatory factor analysis of the Brazilian version of the Drinking Motives Questionnaire-Revised (DMQ-R). Addictive Behaviors, 37, 524-527. doi:10.1016/j.addbeh.2011.11.023.
IBM Corp. (2013). IBM SPSS Statistics for Windows, Version 22.0 [Computer software]. Armonk, NY: IBM Corp.

Kuntsche, E. y Kuntsche, S. (2009). Development and validation of the Drinking Motive Questionnaire Revised Short Form (DMQ-R SF). Journal of Clinical Child and Adolescent Psychology, 38, 899-908. doi:10.1080/15374410903258967.

Kuntsche, E., Wiers, R. W., Janssen, T. y Gmel, G. (2010). Same wording, distinct concepts? Testing differences between expectancies and motives in a mediation model of alcohol outcomes. Experimental and Clinical Psychopharmacology, 18, 436-444. doi:10.1037/a0019724.

Liebregts, N., van der Pol, P., de Graaf, R., Korf, D. J., van den Brink, W. y van Laar, M. (2013). Predicting the transition from frequent cannabis use to cannabis dependence: a three-year prospective study. Drug and Alcohol Dependence, 133, 352-359. doi:10.1016/j.drugalcdep.2013.06.009.

Lloret Irles, D., Morell-Gomis, R., Laguía, A. y Moriano, J.A. (2018). Design and validation of a Cannabis Use Intention Questionnaire (CUIQ) for adolescents. Adicciones, 30, 54-65. doi:10.20882/adicciones.865.

McCabe, S. E. (2002). Gender differences in collegiate risk factors for heavy episodic drinking. Journal of Studies on Alcohol, 63, 49-56.

Meyer, J. P. (2014). Applied Measurement with JMetrik. New York: Taylor \& Francis.

Mezquita, L., Camacho, L., Suso, C., Ortet, G. y Ibáñez, M.I. (2018). Development and validation of the alcohol Expectancy Questionnaire Short Form (EQ-SF). Adicciones, 30, 271-281. doi:10.20882/adicciones.920.

Mezquita, L., Ibáñez, M. I., Moya, J., Villa, H., Arias, B., Fañanás, L. y Ortet, G. (2018). Psychometric Properties of Drinking Motives Questionnaire-Revised (DMQ-R) in Spanish Adolescents. European Journal of Psychological Assessment, 34, 145-153. doi:10.1027/1015-5759/a000319.

Mezquita, L., Ibáñez, M. I., Moya, J., Villa, H. y Ortet, G. (2014). A longitudinal examination of different etiological pathways to alcohol use and misuse. Alcoholism, Clinical E゚ Experimental Research, 38, 1770-1779. doi:10.1111/ acer.12419.

Mezquita, L., Stewart, S. H., Kuntsche, E. y Grant, V. V. (2016). Cross-cultural examination of the five-factor model of drinking motives in Spanish and Canadian undergraduates. Adicciones, 28, 215-220. doi:10.20882/ adicciones.822.

Milfont, T. L. y Fischer, R. (2010). Testing measurement invariance across groups: Applications in cross-cultural research. International Journal of Psychological Research, 3, 111-121. doi:10.21500/20112084.857.

Moitra, E., Christopher, P. P., Anderson, B. J. y Stein, M. D. (2015). Coping-motivated marijuana use correlates with DSM-5 cannabis use disorder and psychological distress among emerging adults. Psychology of Addictive Behaviors, 29, 627-632. doi:10.1037/adb0000083. 
Muñiz, J., Elosua, P. y Hambleton, R. K. (2013). Directrices para la traducción y adaptación de los tests: segunda edición. Psicothema, 25, 151-157. doi:10.7334/psicothema2013.24

OMS (en prensa). The health and social effects of nonmedical cannabis use. Recuperado de goo.gl/ozDftX.

R Core Team (2013). R: A language and environment for statistical computing [Computer Software]. R Foundation for Statistical Computing, Vienna, Austria.

Satorra, A. y Bentler, P. M. (2001). A scaled difference chi-square test statistic for moment structure analysis. Psychometrika, 66, 507-514.

Simons, J. S., C., J. C., Simons, R. M. y Spelman, P. J. (2016). Marijuana consequences in a motivational context: Goal congruence reduces likelihood of taking steps toward change. Addictive Behaviors, 52, 83-90. doi:10.1016/j.addbeh.2015.08.010.

Simons, J. S., Correia, C. J., Carey, K. B. y Borsari, B. E. (1998). Validating a five-factor marijuana motives measure: Relations with use, problems, and alcohol motives. Journal of Counseling Psychology, 45, 265-273. doi:10.1037/0022-0167.45.3.265.

Simons, J. S., Gaher, R. M., Correia, C. J., Hansen, C. L. y Christopher, M. S. (2005). An affective-motivational model of marijuana and alcohol problems among college students. Psychology of Addictive Behaviors, 19, 326-334. doi:10.1037/0893-164X.19.3.326.

Studer, J., Baggio, S., Mohler-Kuo, M., Dermota, P., Daeppen, J.-B. y Gmel, G. (2014). Differential association of drinking motives with alcohol use on weekdays and weekends. Psychology of Addictive Behaviors, 28, 651-658. doi:10.1037/a0035668.

Tu, Y.-K., Gunnell, D. y Gilthorpe, M. S. (2008). Simpson's Paradox, Lord's Paradox, and Suppression Effects are the same phenomenon-the reversal paradox. Emerging Themes in Epidemiology, 5, 2. doi:10.1186/1742-7622-5-2.

UNODC (2015). World drug report 2015. Vienna: Oficina de las Naciones Unidas contra la Droga y el Delito.

Zvolensky, M. J., Vujanovic, A. a, Bernstein, A., Bonn-Miller, M. O., Marshall, E. C. y Leyro, T. M. (2007). Marijuana use motives: A confirmatory test and evaluation among young adult marijuana users. Addictive Behaviors, 32, 3122-3130. doi:10.1016/j.addbeh.2007.06.010. 